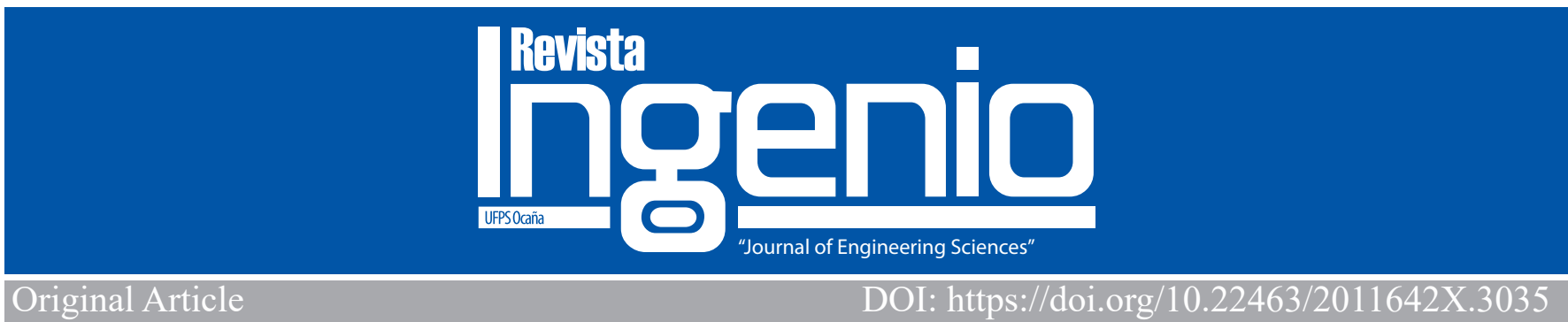

\title{
Desarrollo de un sistema de monitoreo para las variables de temperatura, presión y pH en un biodigestor anaeróbico
}

Development of a monitoring system for temperature, pressure and $\mathrm{pH}$ variables in an Anaerobic Biodigester

MSc. July Andrea Gómez-Camperos ${ }^{1}$, MSc. Fernando Jesús Regino-Ubarnes², MSc. Haidee Yulady-Jaramillo³

${ }^{1}$ Grupo de investigación GINSTI, Universidad Francisco de Paula Santander Ocaña, Colombia, Orcid:https://orcid.org/0000-0003-3894-144X, Email: jagomezc@ufpso.edu.co

${ }^{2}$ Servicio Nacional de Aprendizaje SENA, Colombia, Orcid: https://orcid.org/0000-0003-0414-8654, Email: fjreginou@ufpso.edu.co

${ }^{3}$ Grupo de investigación GINSTI, Universidad Francisco de Paula Santander Ocaña, Colombia, Orcid: https://orcid.org/0000-0002-4185-119X, Email: hyjaramillo@ufpso.edu.co

Cómo citar: J. A. Gómez-Camperos, F. J Regino-Ubarnes \& H. Y. Jaramillo, "Desarrollo de un sistema de monitoreo para las variables de temperatura, presión y pH en un biodigestor anaeróbico”, Revista Ingenio, vol. 19(1), pp. 22-27, 2022.

Fecha de recibido: 11 de mayo de 2021 Fecha aprobación: 25 de octubre de 2021

\section{RESUMEN}

Palabras claves:

En el siguiente documento se presenta un sistema para el mejoramiento del proceso de obtención de energía mediante un biodigestor, se propone que el sistema realice la adquisición de datos de forma automática, permitiendo la visualización de las variables de temperatura, presión y potencial de hidrógeno. La automatización del proceso permite la toma de decisiones en el estudio de la fase de explotación y extracción de gases por medio de la adquisición de datos, sacando el máximo provecho de los residuos biosólidos producto de las actividades agropecuarias. Esta investigación se centra en la elaboración de un sistema de supervisión el cual permite analizar mediante un instrumento virtual las variables de temperatura, presión y $\mathrm{pH}$ involucradas en la adquisición de datos del sistema del Biodigestor, con el fin de nivelar la carga orgánica generada por los componentes de origen agropecuario, la cual generalmente se vierte de forma directa a los causes de agua y en campos a cielo abierto. El instrumento virtual permite la supervisión y el modelamiento de las variables medidas, el estudio de estas puede llevar al mejoramiento de los procesos de biodigestión de biomasa para la generación de gas metano.

Automatización,

Biodigestor, Biomasa.

\section{ABSTRACT}

Key words:

Data acquisition,

Automation, Biodigester, Biomass.
The following document presents a system for the improvement of the process of obtaining energy through a biodigester, it is proposed that the system performs data acquisition automatically, allowing the visualization of the variables of temperature, pressure and hydrogen potential. The automation of the process allows decision making in the study of the exploitation and gas extraction phase by means of data acquisition, making the most of the biosolid waste from agricultural activities. This research is focused on the elaboration of a monitoring system which allows the analysis through a virtual instrument of the temperature, pressure and hydrogen potential variables involved in the data acquisition of the Biodigester system, in order to level the organic load generated by the components of agricultural origin, which is generally discharged directly to water sources and in open fields. The virtual instrument allows the monitoring and modeling of measured variables, the study of which can lead to the improvement of biomass biodigestion processes for methane gas generation.

\section{Introducción}

Es claro que actualmente la generación y el uso de energía influyen tanto en la industria como en la vida cotidiana de los seres humanos [20]. Hoy en día, las energías renovables se presentan como una de las opciones que ha tomado mayor relevancia para contrarrestar el uso de combustibles fósiles [1].

Estas energías renovables que se caracterizan por la utilización de biomasa para la generación se denominan bioenergías, que, para este caso en particular, se refiere al gas generado en reactores mediante la digestión anaerobia de residuos orgánicos. Las bioenergías se muestran como una solución para las necesidades energéticas mundiales con beneficios ambientales, gracias al aprovechamiento de materia de origen orgánico para la producción de metano [2$3]$.

La producción de metano $\left(\mathrm{CH}_{4}\right)$ en el proceso de fermentación de materia orgánica no aprovechada, contribuye a las emisiones de gases de efecto invernadero (GEI). Este subproducto de la descomposición natural de 
la materia orgánica se puede aprovechar si esa se realiza de forma controlada, para esto se propone la utilización de un biodigestor [4-5].

Este trabajo consiste en el desarrollo de un sistema de supervisión de variables que intervienen en el proceso de descomposición controlada de biomasa. Estas variables se miden en un prototipo de un biodigestor a cuál se le supervisa la temperatura, presión y $\mathrm{pH}[6]$.

La transmisión de señales se monitorea por medio de sensores en un instrumento virtual (VI), analizado en LabVIEW, este software especializado para la instrumentación virtual, permite integrar sensores e interfaz gráfica de usuarios (IGU) a través de un sistema de adquisición de datos de temperatura, presión y pH [7].

\section{Metodología}

El proceso de desarrollo del sistema de vigilancia de las variables de temperatura, presión y $\mathrm{pH}$ integradas en el sistema [21], se analizan en un prototipo de un biodigestor anaeróbico, el cual consiste en un contenedor cerrado, en ausencia de oxígeno libre, hermético y sin filtraciones de agua, la materia orgánica a fermentar se coloca en el interior del contenedor en una determinada dilución de agua para que se produzca biogás por fermentación anaeróbica [8].

Las variables de temperatura, presión y $\mathrm{pH}$ son analizadas a traves de la recololecion de datos, existen estudios realizados por distintos autores en donde analizan el Biodigestor Anaeróbico con referencia a distintos tipos de materia organica, temperatura variante, entre otras. Los elementos que influyen en el comportamiento del biodigestor se presentan en la Tabla 1 [9-10].

Tabla 1. Relación de parámetros de un digestor.

\begin{tabular}{ll} 
PARÁMETRO & RANGO ÓPTIMO \\
\hline $\mathrm{pH}$ & $6.8-7.5$ \\
\hline Temperatura $\left({ }^{\circ} \mathrm{C}\right)$ & $30-35$ \\
\hline Tiempo de Retención (días) & $10-25$ \\
\hline Relación C/N & $20-30$ \\
\hline Relación agua/ sólidos & $6-10$ \\
\hline $\begin{array}{l}\text { Presión de diseño (kgf } \\
\left./ \mathrm{cm}^{2}\right)\end{array}$ & 14.0614 \\
\hline
\end{tabular}

Fuente:[12]

- Temperatura y tiempo de retención: el intervalo de temperatura y el lapso de permanencia dentro del biodigestor permiten clasificar la fermentación de la siguiente forma:

o Fermentación mesófila, para un intervalo de temperatura entre 20 y $35^{\circ} \mathrm{C}$ y unos 30 a 40 días de digestión.

o Fermentación termófila, para un intervalo de temperatura de entre 50 y $60^{\circ} \mathrm{C}$ y con más de 8 días de permanencia. Este tipo de fermentos no es el adecuado para las plantas simples.

- Relación C/N: Los materiales de las fermentaciones están compuestos mayoritariamente por carbono (C) y además contienen nitrógeno $(\mathrm{N})$, por lo que se establece la proporción entre ambos $(\mathrm{C} / \mathrm{N})$, que influye en la generación de gas. Una proporción de 20:1 a 30:1 es admisible, aunque el valor ideal es 16; las mezclas de materiales de fermento con elevado porcentaje de nitrógeno con materiales de fermento con elevado porcentaje de carbono generan una gran cantidad de gas. Es preciso mezclar los materiales en porcentajes adecuados con producciones bajas y altas y diferentes tasas de producción para conseguir un buen desempeño de generación de gas durante la fermentación [11].

- Amoniaco: Cobra importancia cuando la biomasa contiene gran cantdad de este. Se aconseja mantener los niveles en el interior de los biodigestores por debajo de $2000 \mathrm{mg} / 1$, por lo que la entrade de la matria prima se debe diluir [11].

- $\mathrm{pH}$ : El potencal de hidrogeno depende de la cantidad de $\mathrm{CO}_{2}$ en el gas, depende tambien de que cantidad de ácidos volátiles y que tan alcalinos son los presentes en la materia prima. Los microorganismos encargados de la producción del biogás no soportan cambios bruscos en el $\mathrm{pH}$, fluctuando entre 6 y 8 . El $\mathrm{pH}$ del lodo de fermentación indica si el proceso de fermentación transcurre sin problemas, y su medición indica el comportamiento de la carga de fermentación dentro del digestor, para la producción de biogás [11].

- Cantidad de humedad de la mezcla: El crecimiento bacteriano y el de otros organismos microscópicos no se da eficazmente cuando la mezcla es demasiado seca, y como consecuencia la cantidad de biogás generado será escasa. Ahora en el caso contrario, cuando hay exceso de agua las bacterias no pueden procesar toda la biomasa y como consecuencia se limita biogás producido. El uso de excrementos y orina humanos, estiércol y residuos agrícolas principalmente como alimento para el digestor debería conducir a una relación biomasa/agua de entre 1:1 y 1:2; y por cada $100 \mathrm{~kg}$ de heces y orina se necesitarán de 100 a 200 litros de agua [11].

- Materiales de origen orgánicos: Las actividades agropecuarias, generan residuos orgánicos que se puedes tratar con microorganismos de forma anaeróbica. Existen diferentes tipos de material orgánico producido por los animales que también se pueden utilizar como base para la elaboración de biogás y bioabono, cuyas características se muestran en la Tabla 2 [11].

El proceso anaeróbico es un mecanismo que se realiza 
en ausencia de oxígeno, en el cual, la materia orgánica es transformada por la acción de los microorganismos en biogás y biocompost; se trata de reacciones bioquímicas en las que participan los microorganismos, una parte de los cuales es completamente oxidada por el carbono convirtiéndose en $\mathrm{CO} 2$, mientras que otra parte es altamente reducida para formar metano [13].

Tabla 2. Valores y características del estiércol.

\begin{tabular}{|c|c|c|c|c|c|c|}
\hline \multirow{2}{*}{ 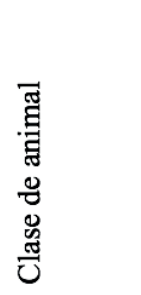 } & \multicolumn{2}{|c|}{ 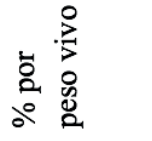 } & \multicolumn{2}{|c|}{ 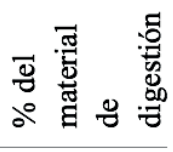 } & \multirow{2}{*}{ 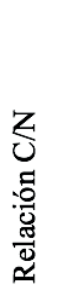 } & \multirow{2}{*}{ 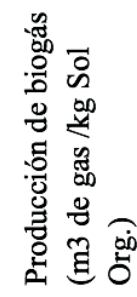 } \\
\hline & 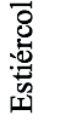 & . & 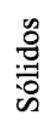 & 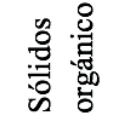 & & \\
\hline Vacunos & 5.0 & 4.0 & $\begin{array}{l}15 \\
- \\
16\end{array}$ & 13 & 20 & 0.250 \\
\hline Cerdos & 2.0 & 3.0 & 16 & 12 & 13 & 0.350 \\
\hline $\begin{array}{l}\text { Caprinos } \\
\text { ovejas }\end{array}$ & 3.0 & 1.5 & 30 & 20 & 30 & 0.200 \\
\hline Caballos & 5.0 & 4.0 & 25 & 15 & 20 & 0.250 \\
\hline $\begin{array}{l}\text { Avícolas, } \\
\text { gallinas }\end{array}$ & 4.5 & 4.5 & 25 & 17 & $\begin{array}{l}5- \\
8\end{array}$ & 0.400 \\
\hline Humanos & 1.0 & 2.0 & 20 & 15 & 8 & 0.300 \\
\hline
\end{tabular}

Fuente:[12].

Este tipo de datos, además de ser útil para el diseño del Biodigestor, se pueden medir a través del sistema de adquisición de datos y su visualización se da mediante instrumento virtual efectuado en LABVIEW, el diseño del biodigestor a analizar se muestra en la Figura 1.
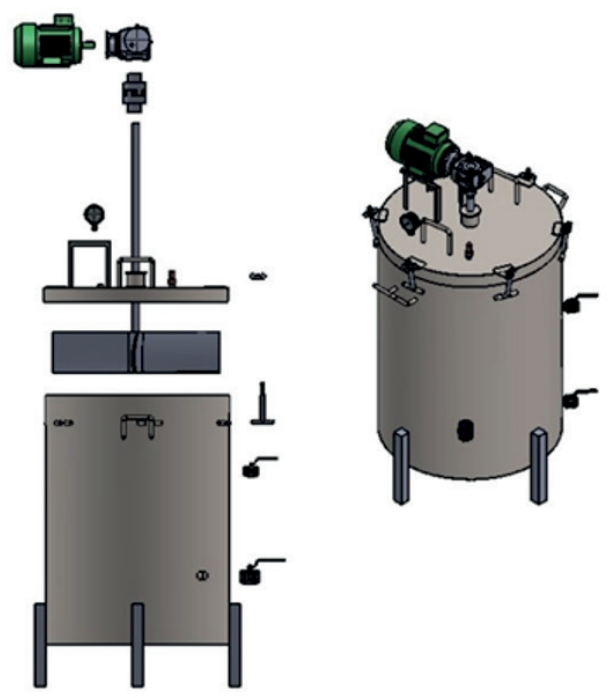

Figura 1. Diseño del Biodigestor.
Tabla 3. Biogás-Elementos que lo integran

\begin{tabular}{|c|c|c|c|}
\hline \multicolumn{4}{|c|}{ Composición del biogás } \\
\hline Elemento & \% Min. & \% Máx. & Prom. \\
\hline Agua & 3.62 & 4.24 & 3.92 \\
\hline $\begin{array}{l}\text { Dióxido de } \\
\text { Carbono }\end{array}$ & 27.5 & 15.01 & 20.85 \\
\hline $\begin{array}{l}\text { Ácido } \\
\text { sulfhídrico }\end{array}$ & 0.1 & 0.01 & 0.18 \\
\hline Metano & 68.78 & 80.65 & 75.05 \\
\hline $\begin{array}{l}\text { Poder cal. } \\
\mathrm{Mj} / \mathrm{m} 3\end{array}$ & 26.84 & 31.12 & 29.17 \\
\hline
\end{tabular}

Fuente: [14]

Para calcular el tamaño del biodigestor se consideran los equipos incluidos, la cantidad de producto que puede contener, la materia prima y las sugerencias para el funcionamiento adecuado considerando el balance estequiométrico de la mezcla a tratar. Para calcular los diseños se deben tener en cuenta el tipo de biomasa para la producción de biogás, la Tabla 3 y 4 muestran el porcentaje de $\mathrm{pH}$ estándar que debe analizar el sistema con referencia al componente de la constitución de biogás y sus distintas características desde el punto de vista de la Cogeneración, para determinar cuanta presión soporta el recipiente [15].

Tabla 4. Biogás - Propiedades.

\begin{tabular}{llllll}
\hline \multicolumn{5}{c}{ Propiedades del biogás y sus componentes. } \\
\hline Propiedades & CH4 & CO2 & $\begin{array}{l}\text { H2- } \\
\text { H2S }\end{array}$ & Otros & $\begin{array}{l}\text { Biogás } \\
60 / 40\end{array}$ \\
\hline Proporciones & $55-$ & $22-44$ & 1 & 3 & 100 \\
& 70 & & & & \\
\hline $\begin{array}{l}\text { Valor } \\
\text { calorífico }\end{array}$ & 860 & - & 2581 & 5258 & 5140 \\
Kcal/m3 & 0 & & & & \\
\hline $\begin{array}{l}\text { Ignición \% en } \\
\text { el aire }\end{array}$ & $5-15$ & - & - & - & $6-12$ \\
\hline $\begin{array}{l}\text { Temperatura } \\
\text { de ignición }{ }^{\circ} \mathrm{C}\end{array}$ & 750 & & & & \\
\hline $\begin{array}{l}\text { Presión critica } \\
\text { en Mpa }\end{array}$ & 4.7 & 7.5 & 1.2 & 8.9 & $7.5-8.9$ \\
\hline $\begin{array}{l}\text { Densidad } \\
\text { nominal en g/1 }\end{array}$ & 0.7 & 1.9 & 0.08 & - & 1.2 \\
\hline $\begin{array}{l}\text { Densidad } \\
\text { relativa }\end{array}$ & 0.55 & 2.5 & 0.07 & 1.2 & 0.83 \\
\hline $\begin{array}{l}\text { Inflamabilidad } \\
\text { vol. en \% aire }\end{array}$ & $5-15$ & - & - & - & $6-12$ \\
\hline
\end{tabular}

Fuente: [14]

Tabla 5. Sensores.

\begin{tabular}{ll}
\hline Descripción & Referencia \\
\hline SENSOR DE PH & SEN0161 \\
\hline TRANSMISOR DE PRESIÓN & EPI 8287 \\
INDUSTRIAL & \\
\hline SENSOR DE TEMPERATURA & TERMO-K \\
\hline
\end{tabular}


Este sistema se le realizara mediante distintos sensores, la Tabla 5 muestran la referencia de cada uno de los sensores integrados en el desarrollo del sistema de supervisión de las variables de temperatura, presión y $\mathrm{pH}$. Los sensores mostrados cumplen con los requisitos mostrados en el diseño del biodigestor, las características de estos se muestran en las Tablas 6,7 y 8 .

- $\quad$ Sensor de temperatura (TERMO K)

Tabla 6. Temperatura.

\begin{tabular}{llll}
\hline & \multicolumn{2}{l}{ Rango De Temperatura } \\
\cline { 2 - 3 } Tipo & & Min ${ }^{\circ} \mathrm{C}$ & $\operatorname{Max}{ }^{\circ} \mathrm{C}$ \\
\hline Termocupla & $\mathrm{K}$ & -80 & 1100
\end{tabular}

Fuente: [16]

- $\quad$ Sensor de $\mathrm{pH}(\mathrm{SEN} 0161)$

Tabla 7. PH.

\begin{tabular}{ll}
\hline Potencia del módulo & $5.00 \mathrm{~V}$ \\
\hline Tamaño de placa de circuito & \multicolumn{2}{l}{$43 \mathrm{~mm} \times 32 \mathrm{~mm}$} \\
\hline Rango de medición de $\mathrm{pH}$ & $0-14$ \\
\hline Medición de temperatura & $0-60^{\circ} \mathrm{C}$ \\
\hline Exactitud & $\begin{array}{l} \pm 0.1 \mathrm{pH}(25 \\
\left.{ }^{\circ} \mathrm{C}\right)\end{array}$ \\
\hline Tiempo de respuesta & $\leq 1 \mathrm{~min}$ \\
\hline Sensor de pH con conector $\mathrm{BNC}$ \\
\hline Interfaz PH2.0 (parche de 3 pies) \\
\hline Potenciómetro de ajuste de ganancia \\
\hline \multicolumn{2}{l}{ LED indicador de encendido }
\end{tabular}

Fuente:[17]

- $\quad$ Transmisor de presión industrial (EPI 8287)

Tabla 8. Presión.

Especificaciones

Señal de salida/

(9...32)VDC

tensión de supply

(9...32)VDC

(9...32)VDC

(15...32)VDC

\begin{tabular}{|c|c|c|}
\hline \multirow[b]{2}{*}{ 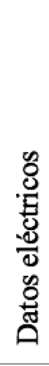 } & Tiempo de subida & $\begin{array}{l}\text { Típ. 1ms/10 } \\
90 \% \text { presión } \\
\text { nominal }\end{array}$ \\
\hline & $\begin{array}{l}\text { Protección contra } \\
\text { inversión de } \\
\text { polaridad, } \\
\text { resistencia a } \\
\text { cortocircuito @ } \\
25^{\circ} \mathrm{C} \text { durante } 5 \mathrm{~min} \text {. }\end{array}$ & $\begin{array}{l}\mathrm{Us}=32 \mathrm{VDC} \\
\mathrm{Us}=28 \mathrm{VDC} \\
\mathrm{Us}=14 \mathrm{VDC}\end{array}$ \\
\hline & $\begin{array}{l}\text { Temperatura del } \\
\text { medio }\end{array}$ & $-40^{\circ} \mathrm{C} \ldots+125^{\circ} \mathrm{C}$ \\
\hline & Temperatura del & $-40^{\circ} \mathrm{C} \ldots+125^{\circ} \mathrm{C}$ \\
\hline
\end{tabular}

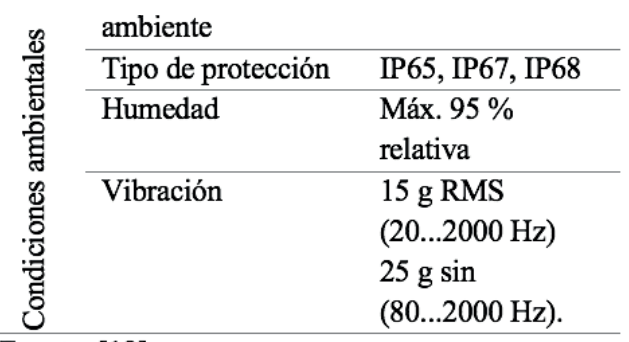

Fuente: [18]

Los sistemas de supervisión de automatización de variables de temperatura, presión y $\mathrm{pH}$ en LabVIEW, permiten el control y monitoreo del sistema, coordinando el funcionamiento de los distintos elementos en la toma de datos, análisis de estos. Este tipo de programas son útiles a la hora de supervisar procesos ya que integran los sensores con los sistemas de adquisición de datos a través de una interfase gráfica de usuario (IGU) denominada "instrumento virtual" (VI). [19]

\section{Resultados y discusión}

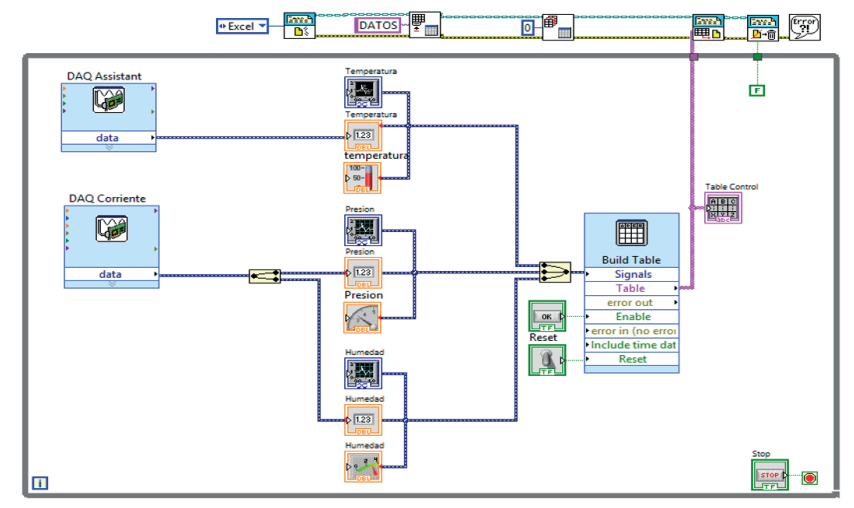

Figura 2. Diagrama de bloques del instrumento virtual con sistema de registro de datos.

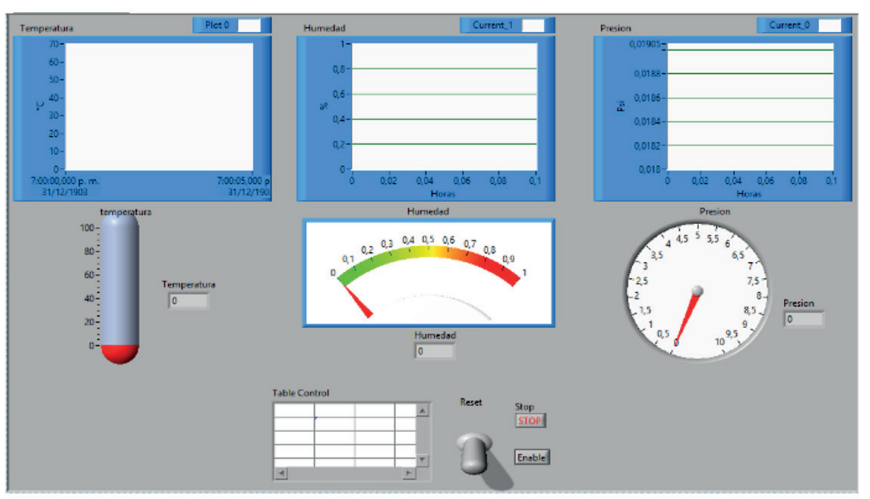

Figura 3. Panel Frontal del instrumento virtual.

En la Figura 2 se muestra el diagrama de bloques del VI, en LabVIEW, permitiendo la adquisición de datos al sistema en dos DAQ de asistencia. El análisis mostrado en el diagrama de bloques permite la recolección de información por medio del formato de EXCEL, en el sistema integrado de 
entradas analogías de temperatura y corriente (presión y pH).

El sistema de supervisión de las variables analizadas se pueden visualizar y monitorizar en el panel frontal mostrado en la Figura 3, en el cual los bloques mostrados en la Figura 2 se esquematizan en el VI de manera automatizada por medio de gráficas, indicadores y medidores en el control del sistema. La temperatura con referencia al tiempo de fermentación varia respecto a la materia orgánica almacena por medio de señales producidas en el sensor implementado, el análisis del sistema de supervisión de la temperatura se muestra en la Figura 4.

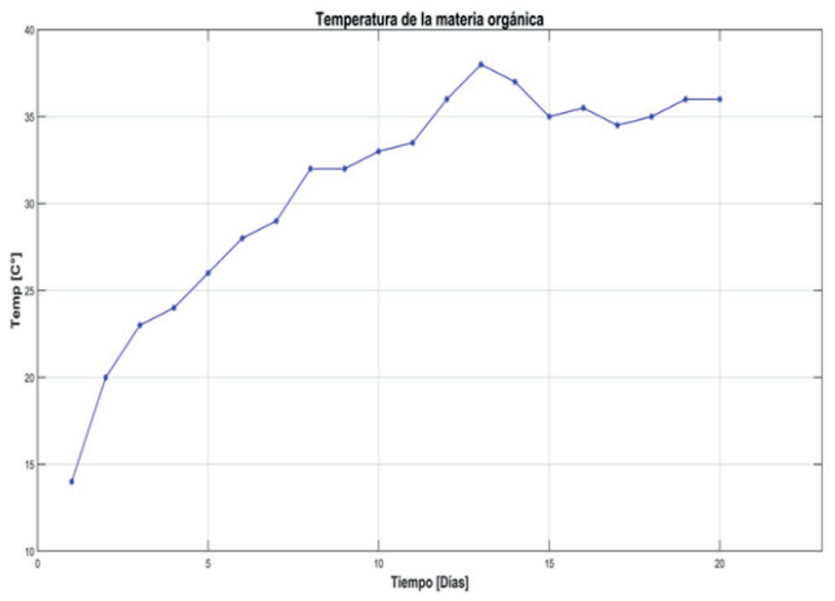

Figura 4. Temperatura VS Tiempo.

La Figura 5, permite observar el comportamiento de la presión generada internamente en el prototipo en un periodo de 20 días, en este tiempo se da la fermentación de la materia orgánica lo que resulta en un aumento de la presión. El comportamiento de la presión en el tiempo que se visualiza en la gráfica es estable a partir de los $9.5 \mathrm{Kpa}$.

El comportamiento del $\mathrm{pH}$ se observa en la Figura 6, los valores de este oscilan entre 6 y 7 , lo que favorece el crecimiento de los microrganismos productores de metano.

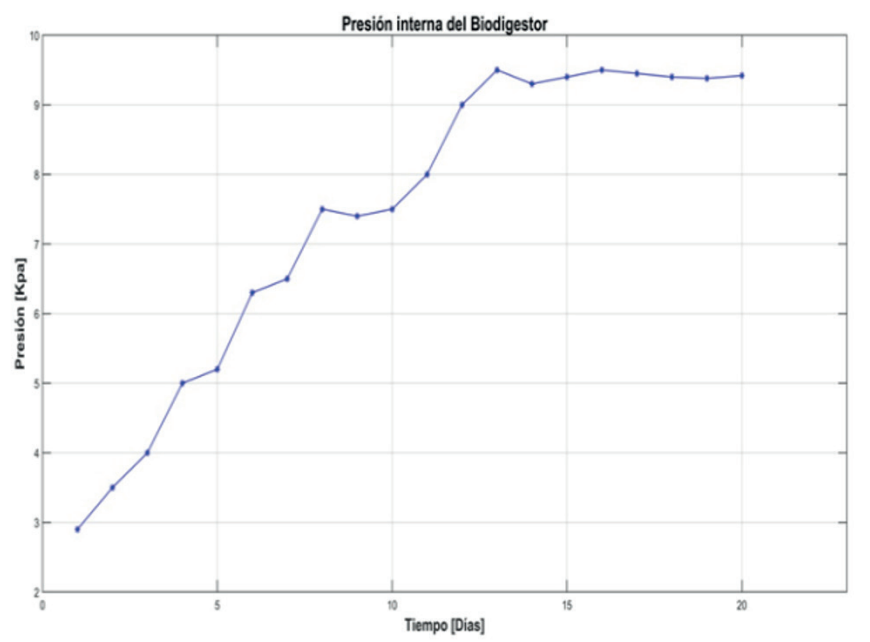

Figura 5. Presión VS Tiempo.

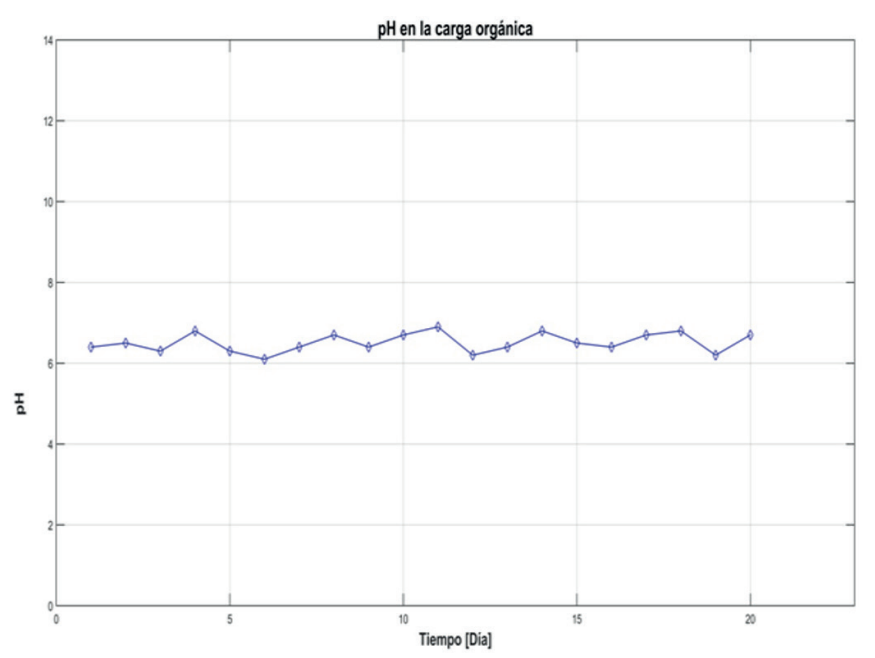

Figura 6. PH VS Tiempo.

\section{Conclusiones}

El sistema de supervisión de variables de temperatura, presión y $\mathrm{pH}$ analizadas en LabVIEW, permiten visualizar las gráficas de cada una de estas variables. Los resultados obtenidos en el desarrollo del proyecto nos permiten concluir que gracias al sistema de monitoreo de variables se reducen costo en la obtención de gases, puesto que al obtener el valor experimental por el programa se puede comparar con los datos estándar del sistema y así extraer el gas combustible optimo del proceso. Otro factor importante por mencionar es el análisis de la producción de la materia prima y el tipo de flujo obtenido al final del proceso reduciendo los efectos ambientales derivados de la actividad agrícola.

El desarrollo del VI en LabVIEW, almacena los datos obtenidos guardándolos en un archivo que es compatible con Microsoft Office Excel, facilitando el tratamiento o procesamiento de los datos y su posterior análisis. Este sistema de supervisión de variables se enfoca en proyectos futuros donde se pueda correlacionar el comportamiento de las variables medidas y su impacto en el desarrollo del proceso de la viabilidad del uso de sensores insertados en el biodigestor, ayudando a monitorear parámetros que son empleados para identificar los tiempos requeridos en la generación de biogás del proceso final.

\section{Referencias}

[1] R. Kigozi, A. O. Aboyade, and E.Muzenda, "Sizing of an anaerobic biodigester for the organic fraction of municipal solid waste," in Lecture Notes in Engineering and Computer Science, vol. 2, pp. 659663,2014

[2] H. Clemens, R. Bailis, A. Nyambane, and V. Ndung, "Energy for Sustainable Development Africa Biogas Partnership Program : A review of clean cooking implementation through market development in East Africa," Energy Sustain. Dev., vol. 46, 
pp. 23-31,2018. Doi: https://doi.org/10.1016/j. esd.2018.05.012

[3] M. E. Gavito et al., "Revista Mexicana de Biodiversidad Ecología, tecnología e innovación para la sustentabilidad : retos y perspectivas en México," Enfermería Univ., vol. 88, pp. 150-160, 2017. Doi:https://doi.org/10.1016/j.rmb.2017.09.001

[4] A. N. Matheri, C. Mbohwa, M. Belaid, and J. C. Ngila, "Design technology for bioenergy conversion of organic fraction of municipal solid waste," Green Energy Technol., (9783319636115), pp.181201,2018

[5] K. Ortegon, "Life cycle engineering in an industrial engineering undergraduate program, from the classroom to the real life of students," in Procedia CIRP, vol. 80, pp. 613-618, 2018.Doi:https://doi. org/10.1016/j.procir.2019.01.011

[6] et al., "Influencia del pH sobre la digestión anaerobia de bio-residuos de origen municipal," Rev. U.D.C.A Actual. Divulg. Científica, vol. 17(2), pp.553-562, 2014.

[7] Regino, F. J., Gómez, J. A., \&amp; Jaramillo, H. Y. (2019). Development of a virtual instrument for the calculation of thermal efficiency under the assumptions of standard cold air in a 3500 cc diesel engine, 2008.Doi: https://doi.org/10.1088/17426596/1409/1/012008

[8] A. Chini et al., "Evaluation of deammonification reactor performance and microrganisms community during treatment of digestate from swine sludge CSTR biodigester," J. Environ. Manage., vol. 246, pp.19-26, 2018. Doi: https://doi.org/10.1016/j. jenvman.2019.05.113

[9] L. F. Calza, C. B. Lima, C. E. C. Nogueira, J. A. C. Siqueira, and R. F.Santos, "Cost assessment of biodigester implementation and biogas-produced energy [Avaliação dos custos de implantação de biodigestores e da energia produzida pelo biogás]," Eng. Agric., vol. 35(6), pp. 990-997, 2015. Doi: https://doi.org/10.1590/1809-4430-Eng.Agric. v35n6p990-997/2015

[10] X.Li,E.Mupondwa, andE. Mupondwa, "Commercial feasibility of an integrated closed-loopethanol-feedlotbiodigester system based on triticale feedstock in Canadian Prairies," Renew. Sustain. Energy Rev., vol. 97, September, pp. 401-413, 2018.

[11] E. C. Medrano, "Alternativas sostenibles para reducción del consumo en los servicios públicos de la vivienda rural en tunja, boyacá," Universidad Santotomas 2017.

[12] H. Hernández and E. Y. Ramírez, "Propuesta para el diseño de un biodigestor para el aprovechamiento de la materia orgánica generada en los frigoríficos de bogotá," Proyecto de grado, Universidad Distrita
Francisco José de Caldas, 2012.

[13] C. A. Chavarín, S. O. Benítez, N. V Limón, and A. G. Ramírez, "Development of anaerobic biodigesters for the treatment of municipal organic waste and biogas generation for use as energy source," in Proceedings of ANES/ASME XXX National Solar Energy Week, 2006, vol. 2006.

[14] C. M. Plugge, "Biogas," Microb. Biotechnol., vol. 10(5), pp. 1128-1130, 2017. Doi: https://doi. org/10.1111/1751-7915.12854

[15] C. V. Garavito and D. R. R. Jiménez, "Diseño y construcción de un prototipo biodigestor tipo mixto para la producción y almacenamiento de gas metano.," 2013

[16] E. J. A. Rodríguez, J. W. M. Ocampo, and C. A. S. Ortega, "Medición de temperatura: sensores termoeléctricos," Sci. Tech., vol. 13(34), pp. 1-6, 2007

[17] J. A. Somoza Chuay, J. E. Eirez Izquierdo, S. Pavoni Oliver, and E. Martín Rodríguez, "Construcción y caracterización de electrodos de Vidrio/ITO/PANI para la medición de pH," RIELAC, vol. XXXV(3), p.p.33-38, 2014

[18] F.A. Vega Reyes, "Transmisor de presión diferencial," J. A. Somoza Chuay, J. E. Eirez Izquierdo, S. Pavoni Oliver, and E. Martín Rodríguez, "Construcción y caracterización de electrodos de Vidrio/ITO/PANI para la medición de pH," RIELAC, vol. XXXV(3), pp.33-38, 2014, 2009.

[19] T. Roy and P. Bhattacharjee, "A LabVIEW-based real-time modeling approach for detection of abnormalities in cancer cells," Gene Reports, vol. 20, p.100788, Sep. 2020

[20] E. N. Flórez-Solano, E. E. Espinel-Blanco, y J. E. Barbosa-Jaimes, "Desarrollo de un soldador por puntos para el laboratorio de proceso de manufactura de la Universidad Francisco de Paula Santander Ocaña", Rev. Ingenio, vol. 16, n. ${ }^{\circ}$ 1, pp. 30-35, ene. 2019.Doi: https://doi.org/10.22463/2011642X.2389

[21] F. O. Díaz-Garcés y F. J. Regino-Ubarnes, "Desarrollo de una estrategia de control basado en ADRC, aplicada a un sistema de bola y viga", Rev. Ingenio, vol. 17, n. ${ }^{\circ} 1$, pp. 15-20, ene. 2020. Doi: https://doi. org/10.22463/2011642X.2391 